\title{
A Fast Simulation Method for the Log-Normal Sum Distribution Using a Hazard Rate Twisting Technique
}

\author{
Nadhir Ben Rached, Fatma Benkhelifa, Mohamed-Slim Alouini, and Raul Tempone \\ Computer, Electrical and Mathematical Science and Engineering (CEMSE) Division \\ King Abdullah University of Science and Technology (KAUST) \\ Thuwal, Makkah Province, Saudi Arabia \\ \{nadhir.benrached, fatma.benkhelifa, slim.alouini, raul.tempone\}@kaust.edu.sa
}

\begin{abstract}
The probability density function of the sum of Lognormally distributed random variables (RVs) is a well-known challenging problem. For instance, an analytical closed-form expression of the Log-normal sum distribution does not exist and is still an open problem. A crude Monte Carlo (MC) simulation is of course an alternative approach. However, this technique is computationally expensive especially when dealing with rare events (i.e. events with very small probabilities). Importance Sampling (IS) is a method that improves the computational efficiency of MC simulations. In this paper, we develop an efficient IS method for the estimation of the Complementary Cumulative Distribution Function (CCDF) of the sum of independent and not identically distributed Log-normal RVs. This technique is based on constructing a sampling distribution via twisting the hazard rate of the original probability measure. Our main result is that the estimation of the CCDF is asymptotically optimal using the proposed IS hazard rate twisting technique. We also offer some selected simulation results illustrating the considerable computational gain of the IS method compared to the naive MC simulation approach.
\end{abstract}

Index Terms-Log-normal sum distribution, Crude Monte Carlo, Rare events, Importance Sampling, Hazard rate twisting.

\section{INTRODUCTION}

The probability density function (PDF) of the sum of Log-normal (SLN) random variables (RVs) is widely used in various applications in digital communication. In cellular mobile communication systems, the total Co-Channel Interference (CCI) power which arises due to the neighboring cells that use the same frequency is generally modeled as SLN RVs [1]. In [2], the outage probability of the signal-to-interferenceplus-noise (SINR) of the intended receiver coincides with the probability that the total CCI power, modeled as a SLN RVs, exceeds a given threshold. The Log-normal distribution is also used to model the large-scale fading in the ultra-wideband (UWB) communications [3], and the weak-to-moderate turbulence channels in free-space optical communications channels [4]. From these examples and many other applications, it is of primordial interest to investigate the statistics of the SLN distribution in order to assess the performance of wireless systems.

A closed-form expression of the Log-normal sum distribution remains unknown. As such, many research efforts

The authors are members of the KAUST Strategic Research Initiative on Uncertainty Quantification in Science and Engineering (SRI-UQ). were carried out to propose various approximations of the sum distribution. These approximations could be divided into three categories. The first category includes techniques where the SLN RVs is approximated by another Log-normal RV. In this category, the most known method is the FentonWilkinson (FW) approximation in [5] where the Log-normal parameters are obtained by moment matching. Another widely used approximation was developed by Schwartz-Yeh (SY) in [6] where the mean and the variance are computed recursively. The SY approximation was further simplified in [7]. Beaulieu and Xie have introduced an optimal Log-normal approximation using a minmax approach [8]. Comparison of the previous cited techniques and other related works could be found in [9][13]. In [8], the numerical PDF of the SLN has shown a clear discrepancy from a Log-normal PDF. That is, all the previously cited works fail to accurately approximate the sum distribution for a certain choice of the Log-normal parameters and the number of summands. A second category of approximations were derived to overcome this failure. For example, in [14], a variant of type IV Pearson distribution was used as an approximation. In [15] and [16], exponential and quadratic functions were respectively developed to approximate the Cumulative Distribution Function (CDF) of the SLN in a Log-normal probability scale. The parameters of these three approximations are based on solving a non linear least square (LS) problem which requires the computation of the empirical CDF of the SLN. Moreover, the dependence of the fitted parameters on the $\mathrm{dB}$ spread, and the number of summands, may result in a higher complexity to use these approximation techniques. Furthermore, the computation of the empirical distribution by numerical computation is expensive because of the slow decay of the tail of the Log-normal PDF. Thus, a third class of methods were introduced to construct efficient numerical integration techniques to approximate the PDF of the SLN. For instance, a steepest-descent integration was used in [17], and in the same framework an approximation based on a Smolyak's algorithm belonging to the family of numerical integration on sparse grid was derived in [18].

In this paper, we develop an efficient Importance Sampling (IS) Monte Carlo (MC) simulation approach to estimate the Complementary Cumulative Distribution Function (CCDF) of the sum of independent and not identically distributed Lognormal RVs. More specifically, a suitable change of the 
probability measure is used to reduce the variance of the MC estimator and hence significantly decrease the number of simulation runs. Due to page limitation, we are not including in the conference version all details of the proofs. However, all details are available in our extended journal version [19] which treats the most general case of sums involving independent RVs with arbitrary distributions. The rest of the paper is organized as follows. In Section II, we present the naive MC simulation and show how it fails. We review also the basic concept of IS methods. Next, we develop in Section III an efficient IS technique and prove its asymptotic optimality criterion. In Section IV, a substantial computational gain of the IS technique is shown through selected simulation results.

\section{MATHEMATICAL BACKGROUND}

\section{A. Naive Monte Carlo Simulation}

Let $X_{1}, X_{2}, \ldots, X_{N}$ be a sequence of independent Lognormal RVs. The PDF of $X_{i}, i=1,2, \ldots, N$, is given by

$$
f_{i}(x)=\frac{1}{\sqrt{2 \pi} \sigma_{i} x} \exp \left(-\frac{\left(\log (x)-\mu_{i}\right)^{2}}{2 \sigma_{i}^{2}}\right), x>0 .
$$

A property of a Log-normal RV is that its logarithm is a Gaussian distribution, that is $Y_{i}=\log \left(X_{i}\right)$ is a normal $\mathrm{RV}$ with parameters $\mu_{i}$ and $\sigma_{i}$. In digital communication, it is frequent that the power signal is expressed in decibel. Hence, it is more convenient to define a Gaussian RV as $Z_{i}=10 \log _{10}\left(X_{i}\right)$ with mean $\mu_{i, d B}$ and variance $\sigma_{i, d B}$. The relations between the two Gaussian random variables $Y_{i}$ and $Z_{i}, i=1, \ldots, N$, are

$$
\begin{aligned}
\mu_{i} & =\xi \mu_{i, d B}, \\
\sigma_{i} & =\xi \sigma_{i, d B},
\end{aligned}
$$

where $\xi=\log (10) / 10$. As mentioned in the previous section, we are interested in estimating the quantity

$$
\alpha=P\left(\sum_{i=1}^{N} X_{i}>\gamma_{t h}\right)=P\left(S_{N}>\gamma_{t h}\right),
$$

for a given threshold $\gamma_{t h}$. The standard technique to estimate $\alpha$ is to use a MC estimator

$$
\hat{\alpha}_{M C}=\frac{1}{M} \sum_{j=1}^{M} \mathbf{1}_{\left(S_{N}\left(\omega_{j}\right)>\gamma_{t h}\right)},
$$

where $M$ is the number of simulation runs, and $\mathbf{1}_{(\cdot)}$ defines the indicator function. The MC simulation is extensively expensive for the estimation of rare events. In fact, from the Central Limit Theorem (CLT), it can be shown that the MC estimation with $10 \%$ relative error needs more than $100 / \alpha$ realizations. Hence, the number of samples to estimate a probability of the order of $10^{-9}$ should be more than $10^{11}$, with an accuracy requirement of $90 \%$.

\section{B. Importance Sampling (IS)}

1) General Approach:

IS is a variance reduction technique which aims to increase the computational efficiency of the naive MC simulation [20]. The general concept of IS is to construct an unbiased estimator of the desired probability with much smaller variance than the naive estimator. In fact, this technique is based on performing a suitable change of the sampling distribution as shown below

$$
\begin{aligned}
\alpha & =\int_{\mathbb{R}^{N}} \mathbf{1}_{\left(S_{N}>\gamma_{t h}\right)} \prod_{i=1}^{N} f_{i}\left(x_{i}\right) d x_{1} d x_{2} \ldots d x_{N} \\
& =\int_{\mathbb{R}^{N}} \mathbf{1}_{\left(S_{N}>\gamma_{t h}\right)} L\left(x_{1}, x_{2}, \ldots, x_{N}\right) \prod_{i=1}^{N} g\left(x_{i}\right) d x_{1} d x_{2} \ldots d x_{N} \\
& =E_{p^{*}}\left[\mathbf{1}_{\left(S_{N}>\gamma_{t h}\right)} L\left(X_{1}, X_{2}, \ldots, X_{N}\right)\right]=E_{p^{*}}\left[T_{\gamma_{t h}}\right]
\end{aligned}
$$

where the expectation is taken with respect to the new probability measure $p^{*}$ under which the PDF of each $X_{i}$ is $g_{i}(\cdot)$, $i=1,2, \ldots, N$, and $L$ is the likelihood ratio defined as

$$
L\left(X_{1}, X_{2}, \ldots, X_{N}\right)=\prod_{i=1}^{N} \frac{f_{i}\left(X_{i}\right)}{g_{i}\left(X_{i}\right)} .
$$

The idea behind this change of measure is to enhance sampling important values, i.e. realizations that satisfy $\left\{S_{N}>\gamma_{t h}\right\}$, which have more impact on the desired probability. Hence, emphasizing that important values are sampled frequently will result in a decrease of the variance of the IS estimator which is defined as

$$
\hat{\alpha}_{I S}=\frac{1}{M} \sum_{i=1}^{M} \mathbf{1}_{\left(S_{N}\left(\omega_{i}\right)>\gamma_{t h}\right)} L\left(X_{1}\left(\omega_{i}\right), \ldots, X_{N}\left(\omega_{i}\right)\right) .
$$

\section{2) Characterizing a Good Change of Measure:}

Generally, it is not obvious how to construct a new probability measure which results in decreasing the variance of the IS estimator and hence increasing the computational efficiency. Besides, it is necessary to define some performance indicators which measure the goodness and the pertinence of the IS estimator. Asymptotic optimality is one of the criterion used to characterize a good change of the probability measure [21]. From the non-negativity of the variance of $T_{\gamma_{t h}}$, we have that

$$
\mathbb{E}_{p^{*}}\left[T_{\gamma_{t h}}^{2}\right] \geq P\left(S_{N}>\gamma_{t h}\right)^{2},
$$

for all probability measure $p^{*}$. By applying the Logarithm function on both sides and using the fact that $\log \left(P\left(S_{N}>\gamma_{t h}\right)\right)<0$, it follows

$$
\frac{\log \left(\mathbb{E}_{p^{*}}\left[T_{\gamma_{t h}}^{2}\right]\right)}{\log \left(P\left(S_{N}>\gamma_{t h}\right)\right)} \leq 2
$$

Hence, we say that $\alpha$ is asymptotically optimally estimated if the above inequality holds with equality as $\gamma_{t h} \rightarrow+\infty$, that is

$$
\lim _{\gamma_{t h} \rightarrow \infty} \frac{\log \left(\mathbb{E}_{p^{*}}\left[T_{\gamma_{t h}}^{2}\right]\right)}{\log \left(P\left(S_{N}>\gamma_{t h}\right)\right)}=2
$$

It is important to note that the naive simulation is not asymptotically optimal for the estimation of $\alpha$ since the ratio in (10) is equal to 1 .

\section{Hazard Rate Twisting of The Log-Normal Sum DisTRIBUTION}

\section{A. Motivation}

In the literature, many IS techniques were developed in order to estimate the value of $\alpha$. The most used IS methods in 
digital communications are conventional IS and improved IS techniques which are based on scaling the variance [22] and shifting the mean [23] of the underlying distribution. Moreover, an exponential twisting method derived from the large deviation theory was developed for the case of distributions with light tails [24]. For instance, the exponential twisting technique was used to estimate the bit-error rate (BER) of direct-detection optical systems employing avalanche photodiode (APD) receivers in [25]. However, the exponential twisting approach cannot work with distributions with heavy tails where the moment generating functions (MGFs) do not exist.

The Log-normal RV is a heavy tailed distribution, i.e it has a heavier tail than any exponential distribution. Therefore, the exponential twisting method could not be used in our setting. Fortunately, an alternative hazard rate twisting algorithm is constructed to deal with the sum of independent and identically distributed (i.i.d) Log-normal RVs and more generally with i.i.d distributions with slow decay [21]. In this work, an extension of [21] to the case of independent and not identically distributed SLN RVs is performed. Our extended version of the present work is in [19] where the hazard rate twisting technique is used to estimate the CCDF of a more general case involving sums of independent arbitrary variates. Let us define the hazard rate $\lambda_{i}(\cdot)$ associated to the random variable $X_{i}, i=1,2, \ldots, N$, as

$$
\lambda_{i}(x)=\frac{f_{i}(x)}{1-F_{i}(x)}, \quad x>0,
$$

where $F_{i}(\cdot)$ is the CDF of $X_{i}, i=1, \ldots, N$. Besides, we define the hazard function as

$$
\Lambda_{i}(x)=\int_{0}^{x} \lambda_{i}(t) d t, \quad x>0 .
$$

For the case of a Log-normal RV, the expressions of the hazard rate and the hazard function are

$$
\begin{aligned}
\lambda_{i}(x) & =\frac{\frac{1}{x \sigma_{i}} \phi\left(\frac{\log (x)-\mu_{i}}{\sigma_{i}}\right)}{Q\left(\frac{\log (x)-\mu_{i}}{\sigma_{i}}\right)}, \\
\Lambda_{i}(x) & =-\log \left(Q\left(\frac{\log (x)-\mu_{i}}{\sigma_{i}}\right)\right),
\end{aligned}
$$

where $\phi(\cdot)$ and $Q(\cdot)$ are the PDF and the CCDF of the standard normal RV respectively. From (11) and (12), the PDF of $X_{i}$ is related to the hazard rate and the hazard function as

$$
f_{i}(x)=\lambda_{i}(x) \exp \left(-\Lambda_{i}(x)\right) .
$$

The change of probability measure is obtained by twisting the hazard rate of the underlying distribution by a quantity $0<\theta<1$

$$
\begin{aligned}
g_{i}(x) & =f_{i, \theta}(x)=(1-\theta) \lambda_{i}(x) \exp \left(-(1-\theta) \Lambda_{i}(x)\right) \\
& =(1-\theta) f_{i}(x) \exp \left(\theta \Lambda_{i}(x)\right) .
\end{aligned}
$$

Consequently, the random variable $T_{\gamma_{t h}}$ has the following expression

$$
T_{\gamma_{t h}}=\frac{1}{(1-\theta)^{N}} \exp \left(-\theta \sum_{i=1}^{N} \Lambda_{i}\left(X_{i}\right)\right) \mathbf{1}_{\left(S_{N}>\gamma_{t h}\right)} .
$$

\section{B. Determination of the Optimal Value of $\theta$}

Generally, an interesting IS change of probability measure for the estimation of rare events is characterized by the property of uniformly bounded likelihood ratio along paths to rare set. This property will result in obtaining an upper bound of the second moment of the RV $T_{\gamma_{t h}}$. Then, the value of the parameter $\theta$ is chosen to minimize that upper bound. This procedure is called a minmax approach and the resulting $\theta$ is called the minmax optimal twisting parameter. More precisely, the minmax procedure for the determination of $\theta$ is divided into two steps. In the first step, we construct an upper bound of $E_{p^{*}}\left(T_{\gamma_{t h}}^{2}\right)$ which is achieved by solving the following maximization problem $(\mathrm{P})$

$$
\begin{array}{ll}
\underset{X_{1}, \ldots, X_{N}}{\max } & L\left(X_{1}, X_{2}, \ldots, X_{N}\right) \\
\text { Subject to } & \sum_{i=1}^{N} X_{i} \geq \gamma_{t h}, \\
& X_{i}>0, \quad i=1, \ldots, N .
\end{array}
$$

Denoting the optimal solution of $(\mathrm{P})$ by $X_{1}^{*}, X_{2}^{*}, \ldots, X_{N}^{*}$, the second step is to choose the optimal value of $\theta$ which minimizes the upper bound of $\mathbb{E}_{p^{*}}\left[T_{\gamma_{t h}}^{2}\right]$. In fact, we have

$$
\begin{aligned}
\mathbb{E}_{p^{*}}\left[T_{\gamma_{t h}}^{2}\right] & =\mathbb{E}_{p^{*}}\left[L^{2}\left(X_{1}, X_{2}, \ldots, X_{N}\right) \mathbf{1}_{\left(S_{N}>\gamma_{t h}\right)}\right] \\
& \leq L^{2}\left(X_{1}^{*}, X_{2}^{*}, \ldots, X_{N}^{*}\right)
\end{aligned}
$$

where the expression of the likelihood ratio using a hazard rate twisting technique is

$$
L\left(X_{1}, X_{2}, \ldots, X_{N}\right)=\frac{1}{(1-\theta)^{N}} \exp \left(-\theta \sum_{i=1}^{N} \Lambda_{i}\left(X_{i}\right)\right)
$$

Consequently, the optimal minmax value of $\theta$ which minimizes the upper bound (18) is

$$
\theta^{*}=1-\frac{N}{\sum_{i=1}^{N} \Lambda_{i}\left(X_{i}^{*}\right)} .
$$

By replacing the hazard function by (14), the expression of the optimal minmax twisting parameter becomes

$$
\theta^{*}=1+\frac{N}{\sum_{i=1}^{N} \log \left(Q\left(\frac{\log \left(X_{i}^{*}\right)-\mu_{i}}{\sigma_{i}}\right)\right)} .
$$

\section{Asymptotic Optimality Property}

Theorem 1. For a sum of independent Log-normal random variables, the quantity of interest $\alpha$ is asymptotically optimally estimated using the hazard rate twisting approach with $\theta$ given in (20).

Proof: The proof is given in details in our companion technical report [19].

D. Generation of Log-Normal Random Variable under the Twisted Distribution

Although the hazard rate twisting of a Log-normal RV $X$ results in an unknown distribution, the generation of $X$ under $f_{\theta}(\cdot)$ can be done using its $\operatorname{CDF} F_{\theta}(\cdot)$. In fact, it is known that 
$F_{\theta}^{-1}(U)$, where $U$ is uniformly distributed RV over $[0,1]$, has the same distribution as $\mathrm{X}$ under the hazard rate twisted PDF $f_{\theta}(\cdot)$. Form (15), the PDF $f_{\theta}(\cdot)$ associated to a Log-normal $\mathrm{RV} X$ with parameter $\mu$ and $\sigma$ could be written as

$$
\begin{aligned}
f_{\theta}(x)= & (1-\theta) \lambda(x) \exp (-(1-\theta) \Lambda(x)) \\
= & \frac{(1-\theta) \phi\left(\frac{\log (x)-\mu}{\sigma}\right)}{\sigma x\left(1-\Phi\left(\frac{\log (x)-\mu}{\sigma}\right)\right)^{\theta}} .
\end{aligned}
$$

Then, the corresponding CDF is given by

$$
F_{\theta}(x)=-\frac{1}{\left(1-\Phi\left(\frac{\log (x)-\mu}{\sigma}\right)\right)^{\theta-1}}+1 .
$$

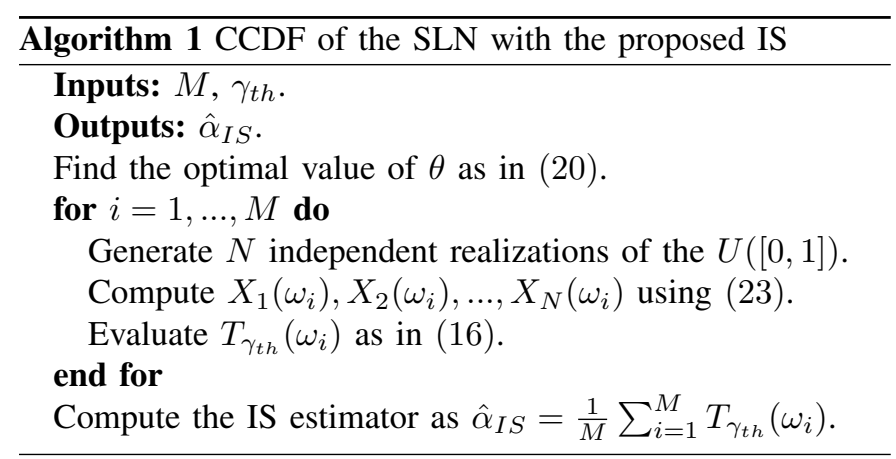

Finally, the CDF inverse $F_{\theta}^{-1}(\cdot)$ can be shown to be given by

$$
F_{\theta}^{-1}(y)=\exp \left(\mu+\sigma \Phi^{-1}\left(1-(1-y)^{-\frac{1}{\theta-1}}\right)\right),
$$

where $\Phi^{-1}(\cdot)$ is the CDF inverse of a standard normal distribution. In Algorithm 1, we present a pseudo-code describing all steps to estimate $\alpha$ by the proposed hazard rate twisting technique.

\section{Simulation Results}

In this section, some selected simulation results are shown to compare the naive MC simulation and the IS simulation technique introduced in the previous section. Two performance metrics will be used to compare between these two approaches. The relative error of the naive MC estimator is defined through the use of the CLT as

$$
\epsilon_{M C}=C \frac{\sqrt{\alpha(1-\alpha)}}{\sqrt{M_{M C}} \alpha},
$$

and the relative error of the IS estimator is given by

$$
\epsilon_{I S}=C \frac{\sqrt{\operatorname{var}_{p^{*}}\left[T_{\gamma_{t h}}\right]}}{\sqrt{M_{I S}} \alpha},
$$

where $C$ is the confidence constant equal to 1.96 (for $95 \%$ confidence interval) in our numerical results, $M_{M C}$ and $M_{I S}$ are the number of samples for the naive $\mathrm{MC}$ and the IS simulations, respectively. For a fixed accuracy requirement, we define the efficiency indicator of the IS technique compared to the naive $\mathrm{MC}$ simulation as

$$
k=\frac{M_{M C}}{M_{I S}}=\frac{\alpha(1-\alpha)}{\operatorname{var}_{p^{*}}\left[T_{\gamma_{t h}}\right]} .
$$

TABLE I

FREQUENCY OF OCCURRENCE ON THE SET $\left\{S_{N} \geq \gamma_{t h}\right\}$ OF THE SUM OF TWO I.I.D. LOG-NORMAL WITH $\mu_{d B}=0 \mathrm{DB}, \sigma_{d B}=6 \mathrm{DB}$ AND $M_{I S}=M_{M C}=10^{5}$

\begin{tabular}{|c|c|c|c|}
\hline Threshold & $\hat{\alpha}_{I S}$ & IS frequency & Naive MC frequency \\
\hline 15 & $1.47 \times 10^{-2}$ & 28603 & 1427 \\
20 & $9.55 \times 10^{-4}$ & 27631 & 99 \\
25 & $3.17 \times 10^{-5}$ & 26484 & 3 \\
30 & $5.8 \times 10^{-7}$ & 26253 & 0 \\
35 & $0.55 \times 10^{-8}$ & 25982 & 0 \\
\hline
\end{tabular}

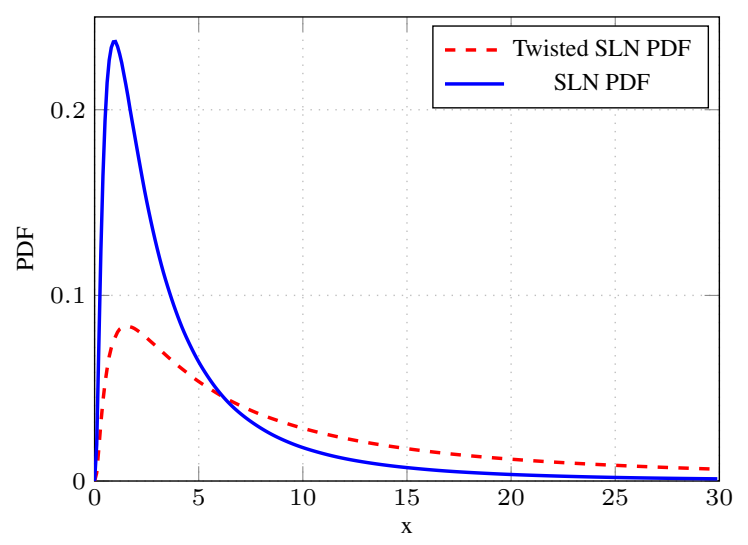

Fig. 1. Twisted and original PDFs of the sum of two i.i.d Log-normal RVs with $\gamma_{t h}=20, \mu_{d B}=0 \mathrm{~dB}$, and $\sigma_{d B}=6 \mathrm{~dB}$.

The larger the efficiency $k$ is, the more samples we need in the naive MC simulation to reach the accuracy given by the proposed IS approach. In other words, the bigger is $k$, the more efficient is the proposed IS technique. The quantity $\alpha$ in (24), (25), and (26) is estimated by the IS estimator $\hat{\alpha}_{I S}$ whereas $\operatorname{var}_{p^{*}}\left[T_{\gamma_{t h}}\right]$ is estimated by the sample variance.

As it was mentioned before, a key characteristic of a good IS technique is to emphasize the sampling of important values, i.e. realizations satisfying $S_{N} \geq \gamma_{t h}$. We define the frequency of occurrence as the number of samples which satisfy $S_{N} \geq \gamma_{t h}$. In our first simulation results, we consider the sum of two i.i.d. Log-normal RVs with mean $\mu_{d B}=0 \mathrm{~dB}$ and standard deviation $\sigma_{d B}=6 \mathrm{~dB}$. In Table I, we have computed the frequency of occurrence using the naive MC simulation and the proposed IS technique, with $M_{M C}=M_{I S}=10^{5}$. Table I exhibits an important feature of the IS change of measure where the frequency of realizations belonging to the rare set $\left\{S_{N} \geq \gamma_{t h}\right\}$ is almost constant as we increase the threshold. On the other hand, the failure of sampling under the original SLN distribution is clear through its inability to construct realizations in the rare sets. To illustrate this statement, we plotted in Fig. 1 the twisted against the original SLN distributions for a fixed threshold $\gamma_{t h}=20$. Clearly, we see that the events which exceed the given threshold are more likely to occur under the twisted PDF than under the original distribution.

In Fig.2, the CCDF is presented using both the naive $\mathrm{MC}$ simulation and our IS simulation technique. The inefficiency of the naive simulation is clear from Fig. 2. In fact, a remarkable oscillatory behavior of the naive MC technique is observed using a number of samples $M_{M C}=10^{6}$ for $\gamma_{t h} \geq 25$ $\mathrm{dB}$. Besides, as we increase the threshold, the naive MC 


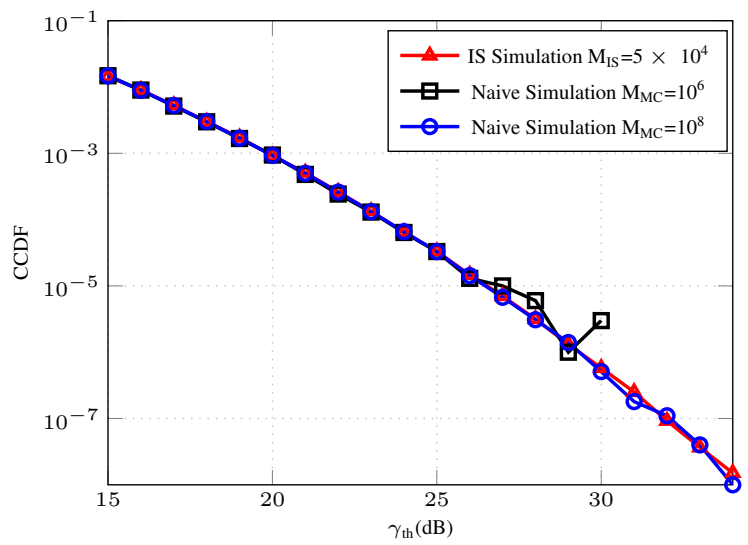

Fig. 2. CCDF of the sum of two i.i.d Log-normal RVs with $\mu_{d B}=0 \mathrm{~dB}$, and $\sigma_{d B}=6 \mathrm{~dB}$

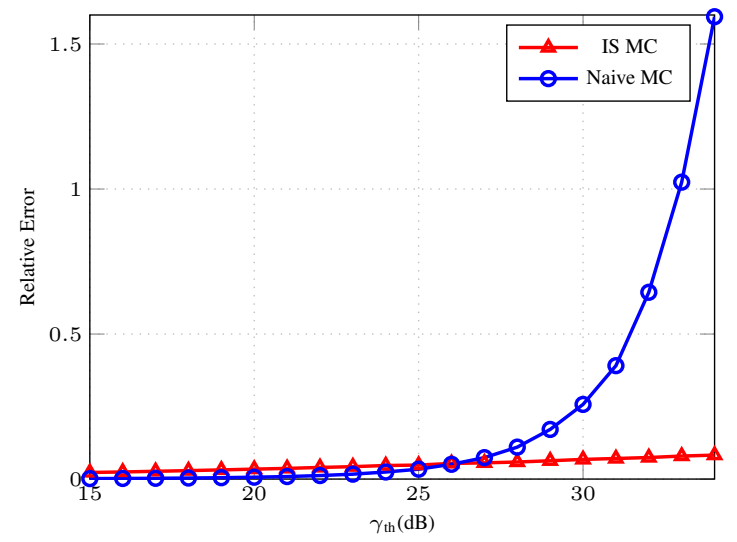

Fig. 3. Relative error of the sum of two i.i.d Log-normal RVs with $\mu_{d B}=0$ $\mathrm{dB}, \sigma_{d B}=6 \mathrm{~dB}, M_{M C}=10^{8}$, and $M_{I S}=5 \times 10^{4}$.

simulation estimates the probability of interest by zero. Indeed, more samples are required in order to overcome this failure and to get a good approximation of the CCDF. The naive technique with $M_{M C}=10^{8}$ is also presented in Fig.2 and is compared to the IS simulation. We point out that both methods coincide and we have a good approximation of the CCDF up to a probability of order of $10^{-6}$. Then, an oscillation of the tail of the CCDF using the naive MC approach is observed, whereas IS technique gives a smooth curve. Moreover, our IS technique gives a more accurate result using a less number of samples $5 \times 10^{4}$, in contrast with $10^{8}$ samples used in the naive simulation. In order to confirm the previous statement, we need to analyze the relative error given by both techniques.

In Fig.3, we plotted the relative error of the naive and the IS simulations in function of the threshold. We point out a slow variation of the relative error of the naive MC simulation for $\gamma_{t h}<25$, then a very rapid increase is observed as we increase the threshold. In fact, in the first region the number of samples is sufficient to guarantee an accurate approximation, whereas in the second region the naive simulation fails to well estimate the CCDF and hence substantial samples are required to ensure a good accuracy, i.e much more than $10^{8}$ realizations. On the other hand, the IS technique shows an interesting result in Fig. 3 where the variation of its relative error is extremely slow compared to the naive simulation. Consequently, with $M_{I S}$ much smaller than $M_{M C}$, our IS approach approximates

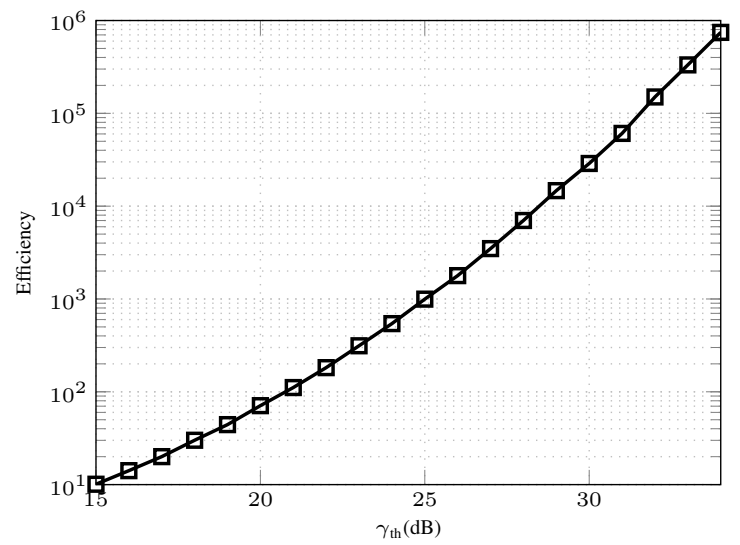

Fig. 4. Efficiency indicator of the sum of two i.i.d Log-normal RVs with $\mu_{d B}=0 \mathrm{~dB}$, and $\sigma_{d B}=6 \mathrm{~dB}$.

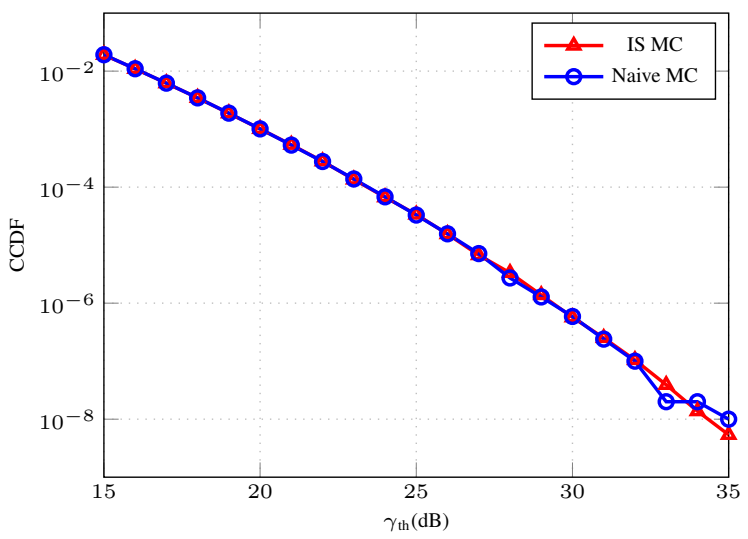

Fig. 5. CCDF of the sum of four independent Log-normal RVs with $\mu_{1, d B}=$ $\mu_{2, d B}=\mu_{3, d B}=\mu_{4, d B}=0 \mathrm{~dB}, \sigma_{1, d B}=\sigma_{2, d B}=4 \mathrm{~dB}, \sigma_{3, d B}=$ $\sigma_{4, d B}=6 \mathrm{~dB}, M_{M C}=10^{8}$, and $M_{I S}=5 \times 10^{5}$.

the CCDF more efficiently than the naive simulation.

In Fig.4, we plotted the efficiency $k$ in function of the threshold. From this figure, we deduce that the efficiency is increasing rapidly, almost exponentially. Hence, the more we increase the threshold the more efficient is our IS technique. For instance, for $\gamma_{t h}=34 \mathrm{~dB}$, we need to sample approximately $M_{M C}=10^{6} \times M_{I S}$ naive MC realizations in order to reach the relative accuracy given by the IS approach. Besides, Fig. 4 illustrates also that the proposed IS technique is more efficient for the considered range of probability, i.e $k$ is always bigger than 1 .

Generally, practical applications where the Log-normal components have different mean values and different standard deviations occur more frequently than the i.i.d case. For instance, the mean and the standard deviation of each CCI power depend on the distance between the base stations and the considered mobile station, the antenna patterns, and the shadowing environment. Consequently, considering the sum of independent and not identically distributed SLN describes more accurately the real statistics of the total received power. The second simulation results is to perform our comparison between the naive MC and the IS simulations for the case of independent and not identically distributed SLN variates. We consider the sum of four independent Log-normal RVs with $\mu_{1, d B}=\mu_{2, d B}=\mu_{3, d B}=\mu_{4, d B}=0 \mathrm{~dB}, \sigma_{1, d B}=\sigma_{2, d B}=4$ 


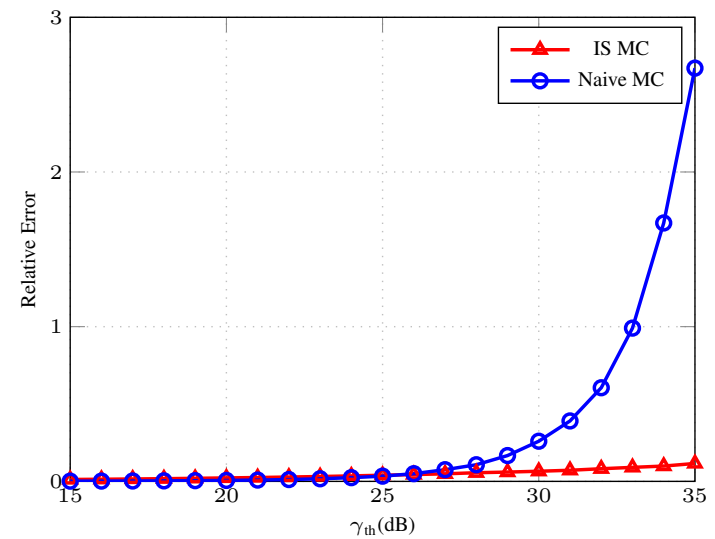

Fig. 6. Relative error of the sum of four independent Log-normal RVs with $\mu_{1, d B}=\mu_{2, d B}=\mu_{3, d B}=\mu_{4, d B}=0 \mathrm{~dB}, \sigma_{1, d B}=\sigma_{2, d B}=4 \mathrm{~dB}$, $\sigma_{3, d B}=\sigma_{4, d B}=6 \mathrm{~dB}, M_{M C}=10^{8}$, and $M_{I S}=5 \times 10^{5}$.

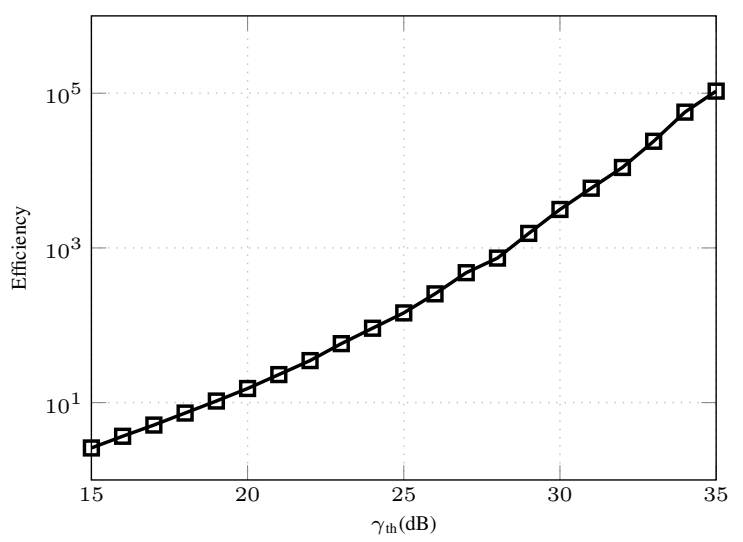

Fig. 7. Efficiency indicator of the sum of four independent Log-normal RVs with $\mu_{1, d B}=\mu_{2, d B}=\mu_{3, d B}=\mu_{4, d B}=0 \mathrm{~dB}, \sigma_{1, d B}=\sigma_{2, d B}=4 \mathrm{~dB}$, and $\sigma_{3, d B}=\sigma_{4, d B}=6 \mathrm{~dB}$.

$\mathrm{dB}$, and $\sigma_{3, d B}=\sigma_{4, d B}=6 \mathrm{~dB}$. In Fig.5, Fig.6, and Fig.7, we plotted the CCDF, the relative error, and the efficiency, respectively. We note that in this case also, the proposed IS technique gives an accurate and efficient approximation of the $\mathrm{CCDF}$ and results in a substantial computational gain.

\section{CONCLUSIONS}

In this paper, we developed an IS technique for the approximation of the CCDF of the sum of independent and not necessarily identically distributed Log-normal RVs. We have shown that the hazard rate twisting of each component of the SLN RVs with an optimal twisting parameter $\theta^{*}$ results in an asymptotic optimal estimator. Numerical simulations showed that the proposed IS method can reach the same accuracy as the naive MC simulation with a substantial computational gain. This alternative efficient technique to the naive MC simulation could serve also as a benchmark for future approximations of the SLN distribution. Moreover, it can be used to decrease the complexity of any approach whose parameters are determined through a curve fitting of data points coming from the exact empirical distribution.

\section{REFERENCES}

[1] G. L. Stüber, Principles of Mobile Communication (2Nd Ed.). Norwell, MA, USA: Kluwer Academic Publishers, 2001.
[2] S. Ben Slimane, "Bounds on the distribution of a sum of independent Lognormal random variables," IEEE Transactions on Communications, vol. 49, no. 6, Jun. 2001.

[3] M. Ghavami, R. Kohno, and L. Michael, Ultra Wideband Signals and Systems in Communication Engineering. Chichester: Wiley, 2004.

[4] S. M. Navidpour, M. Uysal, and M. Kavehrad, "BER performance of free-space optical transmission with spatial diversity." IEEE Transactions on Wireless Communications, vol. 6, no. 8, pp. 2813-2819, Aug. 2007.

[5] L. Fenton, "The sum of Log-normal probability distributions in scatter transmission systems." IRE Transactions on Communications Systems, vol. 8, no. 1, pp. 57-67, 1960.

[6] S. C. Schwartz and Y. S. Yeh, "On the distribution function and moments of power sums with Lognormal component." The Bell Systems Technical Journal, 1982.

[7] C.-L. Ho, "Calculating the mean and variance of power sums with two Log-normal components," IEEE Transactions on Vehicular Technology, vol. 44, no. 4, pp. 756-762, Nov. 1995.

[8] N. Beaulieu and Q. Xie, "An optimal Lognormal approximation to Lognormal sum distributions," IEEE Transactions on Vehicular Technology, vol. 53, no. 2, pp. 479-489, Mar. 2004.

[9] N. Beaulieu, A. Abu-Dayya, and P. McLane, "Comparison of methods of computing Lognormal sum distributions and outages for digital wireless applications," in IEEE International Conference on Communications (ICC '94)., New Orleans, Louisiana, US, May. 1994.

[10] P. Cardieri and T. Rappaport, "Statistics of the sum of Lognormal variables in wireless communications," in IEEE 51st Vehicular Technology Conference Proceedings (VTC'2000-Spring), Tokyo, Japan, May. 2000.

[11] N. Beaulieu, A. Abu-Dayya, and P. McLane, "Estimating the distribution of a sum of independent Lognormal random variables," IEEE Transactions on Communications, vol. 43, no. 12, pp. 2869-2873, Dec. 1995.

[12] J. Filho, P. Cardieri, and M. Yacoub, "Simple accurate Lognormal approximation to Lognormal sums," Electronics Letters, vol. 41, no. 18 , pp. 1016-1017, Sept. 2005.

[13] J. Filho, M. Yacoub, and P. Cardieri, "Highly accurate range-adaptive Lognormal approximation to Lognormal sum distributions," Electronics Letters, vol. 42, no. 6, pp. 361-363, Mar. 2006.

[14] S. Chen, H. Nie, and B. Ayers-Glassey, "Lognormal sum approximation with a variant of type IV Pearson distribution," IEEE Communications Letters, vol. 12, no. 9, pp. 630-632, Sept. 2008.

[15] N. Beaulieu and F. Rajwani, "Highly accurate simple closed-form approximations to Lognormal sum distributions and densities," IEEE Communications Letters, vol. 8, no. 12, pp. 709-711, Dec. 2004.

[16] L. Zhao and J. Ding, "A strict approach to approximating Lognormal sum distributions," in Canadian Conference on Electrical and Computer Engineering (CCECE '2006), Ottawa, Canada, May. 2006.

[17] D. Senaratne and C. Tellambura, "Numerical computation of the Lognormal sum distribution," in IEEE Global Telecommunications Conference (GLOBECOM'2009), Honolulu, Hawaii, US, Nov. 2009.

[18] M. Di Renzo, L. Imbriglio, F. Graziosi, and F. Santucci, "Smolyak's algorithm: A simple and accurate framework for the analysis of correlated Log-normal power-sums," IEEE Communications Letters, vol. 13, no. 9 , pp. $673-675$, Sept. 2009

[19] N. Ben Rached, F. Benkhelifa, A. Kammoun, M.-S. Alouini, and R. Tempone, "Additional results on the hazard rate twisting-based simulation approach," Submitted to IEEE Transactions on Information Theory, 2014. [Online]. Available: http://sri-uq.kaust.edu.sa/Pages/ Page_BenRached_Benkhelifa_Kammoun_Alouini_Tempone_1.aspx

[20] J. A. Bucklew, Introduction to Rare Event Simulation, ser. Springer series in statistics. New York: Springer, 2004.

[21] S. Juneja and P. Shahabuddin, "Simulating heavy tailed processes using delayed hazard rate twisting," ACM Trans. Model. Comput. Simul., vol. 12, no. 2, pp. 94-118, Apr. 2002.

[22] D. Lu and K. Yao, "Improved importance sampling technique for efficient simulation of digital communication systems," IEEE Journal on Selected Areas in Communications, vol. 6, no. 1, pp. 67-75, Jan. 1988.

[23] C. Jeruchim, P. Hahn, K. Smyntek, and R. Ray, "An experimental investigation of conventional and efficient importance sampling," IEEE Transactions on Communications, vol. 37, no. 6, pp. 578-587, Jun. 1989.

[24] J. Sadowsky, "On the optimality and stability of exponential twisting in Monte Carlo estimation," IEEE Transactions on Information Theory, vol. 39, no. 1, pp. 119-128, Jan. 1993.

[25] K. Ben Letaief, "Performance analysis of digital lightwave systems using efficient computer simulation techniques," IEEE Transactions on Communications, vol. 43, no. 234, pp. 240-251, Feb. 1995. 